\title{
Techniques for calibrating spindles with nanometer error motion
}

\author{
Robert Grejda ${ }^{\mathrm{a}}$, Eric Marsh ${ }^{\mathrm{b}, *}$, Ryan Vallance $^{\mathrm{c}}$ \\ a Corning Tropel Corporation, 60 O'Connor Road, Fairport, NY 14450, USA \\ b The Pennsylvania State University, 21 Reber Building, University Park, PA 16802, USA \\ c The George Washington University, 738 Phillips Hall, 801 22nd Street N.W., Washington, DC 20052, USA
}

Received 10 December 2001; received in revised form 16 February 2004; accepted 17 May 2004

Available online 28 July 2004

\begin{abstract}
This work demonstrates techniques that advance the standard practice in spindle metrology and enable five degree-of-freedom calibration of precision spindles with nanometer-level error motion. Several improvements are described in this paper: (1) an improved implementation of Donaldson and Estler reversal that eliminates moving and realigning the displacement sensor, (2) frequency domain low-pass filtering of data to remove spectral content without distortion, (3) robust removal of low frequency components caused by thermal drift and fluctuations in air bearing supply pressure, and (4) three-dimensional display of the synchronous error motion in the radial and axial directions. Example measurements demonstrate the repeatability and reproducibility of the techniques. Furthermore, synchronous radial error motion of an air bearing spindle calibrated by multi-step, master artifact, and master axis techniques agree within $1 \mathrm{~nm}$.
\end{abstract}

(C) 2004 Elsevier Inc. All rights reserved.

Keywords: Spindle metrology; Spindle calibration; Donaldson reversal; Estler reversal; Error separation

\section{Introduction}

The performance of precision spindles is a significant contributor to the overall accuracy of a precision machine or instrument [1-3], and precision spindle designs are now fairly mature and commercially available. For years, the error motion of the highest precision spindles was essentially too small to reliably quantify because the measurement uncertainty exceeded the measurand (a few nanometers for the best spindles). However, improvements in sensors, instrumentation, data acquisition, signal processing, and structural design are closing this gap and enabling low uncertainty spindle metrology. This article demonstrates that spindles with error motion as small as a few nanometers can now be calibrated when these widely available improvements are combined with new spindle metrology-specific developments.

The ANSI/ASME B89.3.4M Axes of Rotation: Methods for Specifying and Testing Standard defines the language of spindle metrology and the reader is referred to the standard for concise, complete and rigorous definitions of the relevant terms [4]. In accordance with this standard, the spindle metrologist must unambiguously specify the imperfections in a spindle's axis of rotation. The spindle rotor is treated as

\footnotetext{
${ }^{*}$ Corresponding author. Tel.: +1-814-865-5242; fax: +1-814-865-2986

E-mail address: emarsh@psu.edu (E. Marsh).
}

a rigid body with six degrees of freedom and ideally $1^{\circ}$ of motion (rotation about the $Z$-axis, by convention). Any motion in the remaining five degrees of freedom is error motion and is quantified and described with techniques and terms from the B89.3.4M standard. This motion is reported by its radial ( $X$ and $Y$ directions at a specified location), axial (along the $Z$-axis), and tilt components (rotation about the $X$ and $Y$ axes). The specific axial location of radial measurements must be reported since tilt components influence radial error motion.

In a complete spindle calibration all five degrees of freedom of error motion are measured as a function of the spindle's angular position. Some applications, such as a lathe with fixed sensitive direction, only require that three degrees of freedom be measured to fully characterize the relevant error motion. The B89.3.4M standard illustrates a number of experimental configurations for measuring the relevant components of error motion.

Fig. 1 illustrates one measurement configuration that records five displacements $\left(X_{1}, Y_{1}, X_{2}, Y_{2}\right.$, and axial) of two spherical artifacts during the revolutions of a spindle; the exact number of revolutions is not rigidly prescribed by B89.3.4M. In such a measurement, the recorded displacements result from both the spindle error motion and imperfections in the artifact geometry (as well as displacements due to thermal drift or other changes in operating condi- 


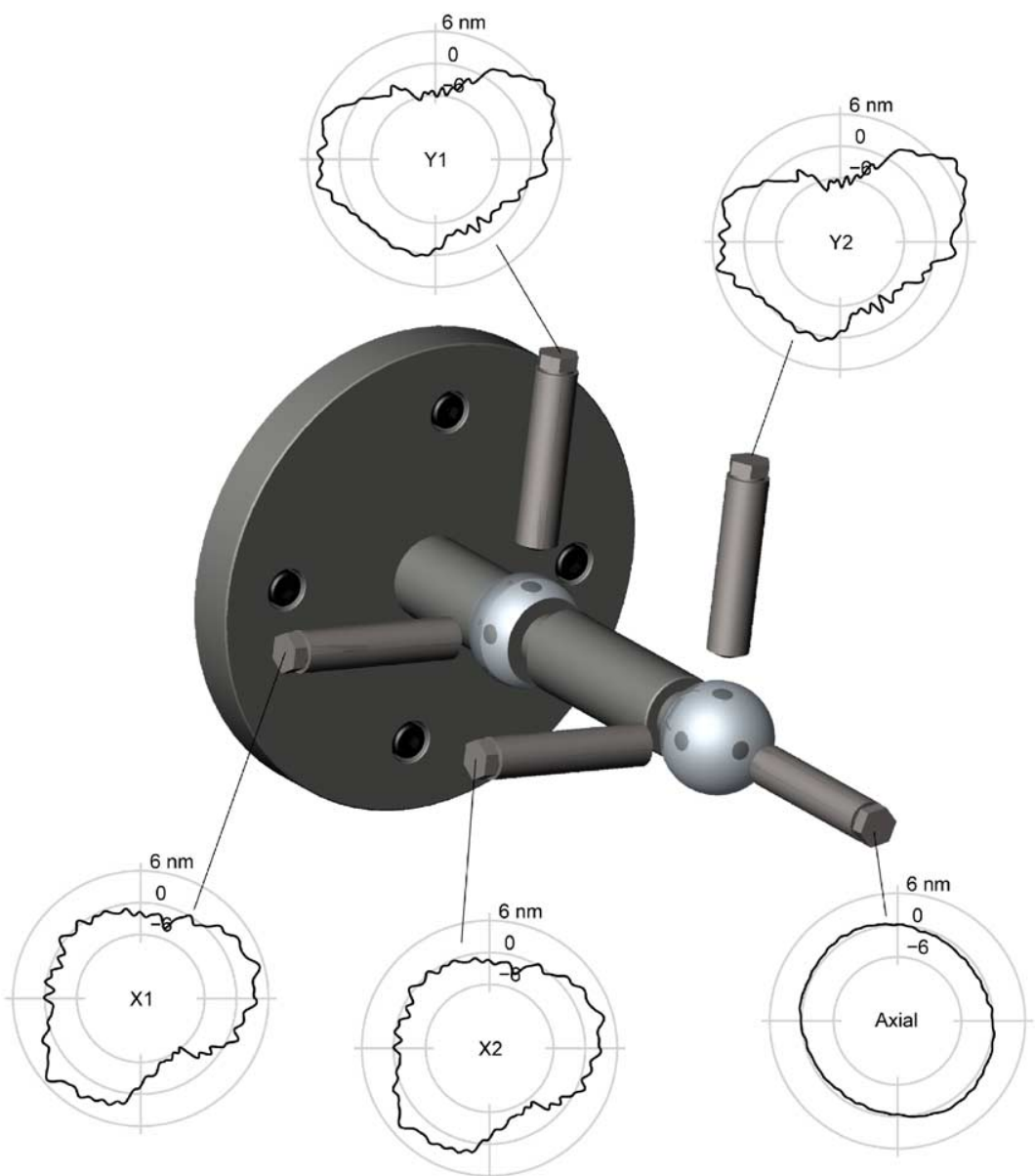

Fig. 1. Double ball artifact and capacitance probes for five degree-of-freedom measurements.

tions). Several techniques can separate spindle error motion from artifact form errors, including Donaldson reversal, Estler reversal, multi-step, and multi-gage methods [5-9].

A second measurement configuration, having compelling advantages as described by Liebers et al. [10], replaces the spherical artifacts with a second, precision spindle called the "master axis". The rotor of the master axis spindle rotates with the rotor of the spindle under test, just as an artifact does in traditional measurements; however, the master axis stator is restrained from rotation about the $Z$-axis. The re-
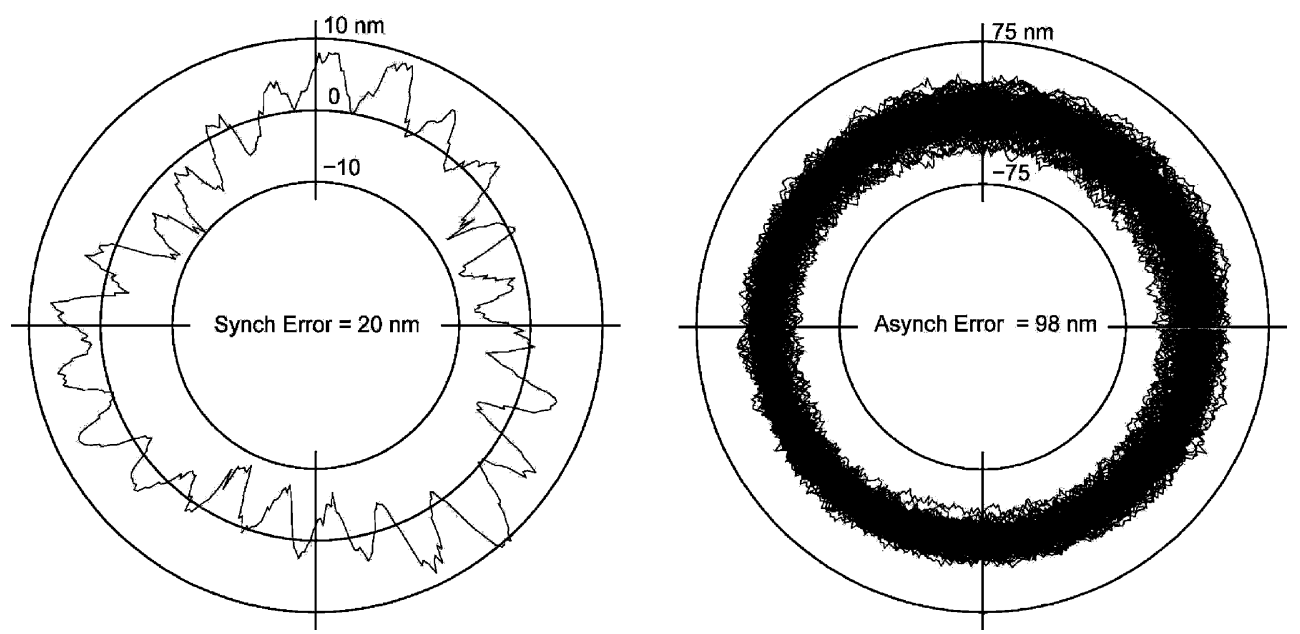

Fig. 2. Synchronous and asynchronous radial error motion of a ball bearing hard disk drive spindle over 128 revolutions. 
straint, which must not constrain the five degrees of freedom of error motion, is effectively realized with a lapped steel sphere in contact with a gage block mounted on the stator of the master axis spindle [11]. Displacement sensors target the non-rotating master axis stator and measure the combined error motion of both spindles. Donaldson reversal or other error separation techniques are used to separate the master axis error motion from that of the spindle under test.

Total error motion, which is the difference between runout and artifact form error, is traditionally illustrated as a function of the spindle's angular position using a polar plot. The mean contour of the total error motion is referred to as average or synchronous error motion, which recently emerged as an equivalent but more descriptive term since average error motion is synchronized to the rotation. The difference between the total error motion and the synchronous error motion is then referred to as asynchronous error motion, since it is not synchronized to the rotation. For example, Fig. 2 shows polar plots of the synchronous and asynchronous components of radial error motion from 128 revolutions of a ball bearing spindle from a hard disk drive. The synchronous error, given as $20 \mathrm{~nm}$ in Fig. 2, is the difference between the maximum and minimum values on the synchronous error motion profile.

\section{Background}

The metrology of machine tools and their components has roots back to the early 1900s. Scheslinger was the first to establish specific tests for qualifying the performance of machine tools and key components such as spindles [12]. Scheslinger's tests of a machine tool spindle used a mechanical displacement indicator on the rotor to measure the total indicated reading (TIR), which is now recognized to be the superposition of the form error of the measured surface and the spindle error motion.

Tlusty and Bryan are considered the founders of modern spindle metrology for developing complete methods for accurately quantifying spindle performance and presenting the results in the intuitive form of polar plots [1,13]. Donaldson's contribution of a robust error separation (reversal) technique in 1972 made possible spindle error motion measurements of accuracy limited only by sensors, data acquisition, structural design and technique [5]. During this period, Bryan, Clouser and Holland's 1967 paper was the basis for a new document published by the International Institution for Production Engineering Research (CIRP) in 1975 and again in 1976 on unifying the terminology of axes of rotation $[14,15]$. These papers became the basis of the B89.3.4M standard that was eventually published in 1985 . In the years that followed, a rich body of work has been published with each successive article further reducing the uncertainty in spindle metrology through clever developments of hardware and technique.
For example, digital processing of spindle measurements was introduced by Vanherck and Peters in 1973, and became widespread in the 1980s as rotary encoders and resolvers appeared more frequently on precision machine tools [16]. In practice, spindle speed fluctuations necessitate accurate angular triggering to properly synchronize data from successive revolutions. Researchers like Arora et al. used resolvers to monitor the phase of artifact eccentricity and efficiently remove it [17]. Murthy et al. used digital notch filters tuned to the spindle rotation rate as a method to remove artifact eccentricity [18].

One of the most important advances in spindle metrology since the work by Bryan and Tlusty was the refinement of capacitance sensors with high sensitivity and resolution. An excellent background on capacitive sensors is available from Foldvari and Lion [19]. By the late 1980s, researchers developed nanometer-resolution sensors that could be applied to the challenge of measuring precision spindle error motion [20]. Chapman designed and built a spindle analyzer capable of measuring radial, axial, and tilt error motion with a resolution of $5 \mathrm{~nm}$ [21]. Hansen introduced a general-purpose instrument in 1988 using the now popular double sphere artifact for complete calibrations [22].

Other developments have been thoughtfully researched, including alternatives to the Donaldson and Estler reversal methods for separating spindle error motion from artifact form error. These alternatives include multi-position techniques where measurements are recorded with displacement sensors at multiple angular locations and multi-probe methods where measurements are recorded from multiple sensors simultaneously. Whitehouse pointed out that the multi-position and multi-probe methods are insensitive to (i.e., do not accurately detect) certain harmonics of the error motion depending on the number of gages, positioning of the gages, or steps used in acquiring the data [23]. Donaldson and Estler reversal are widely preferred since they completely separate all harmonics of error motion and artifact form. Regardless of the error separation scheme used in a particular spindle calibration, the literature and experience show that the accuracy of all methods is adversely affected by imperfectly re-positioned gages or by using multiple gages of unequal sensitivity. Therefore great care is required when reorienting a displacement sensor and reversing the spindle or artifact.

\section{Experimental techniques}

Despite continuous improvements in metrology, it remains formidable to calibrate the highest precision spindles with nanometer error motion. In this section we describe our experimental techniques that are subsequently shown to repeatably and reproducibly calibrate precision spindles with error motion at the nanometer level. These techniques are described in the context of externally pressurized air bearing spindles commonly found in diamond turning, precision grinding, and 

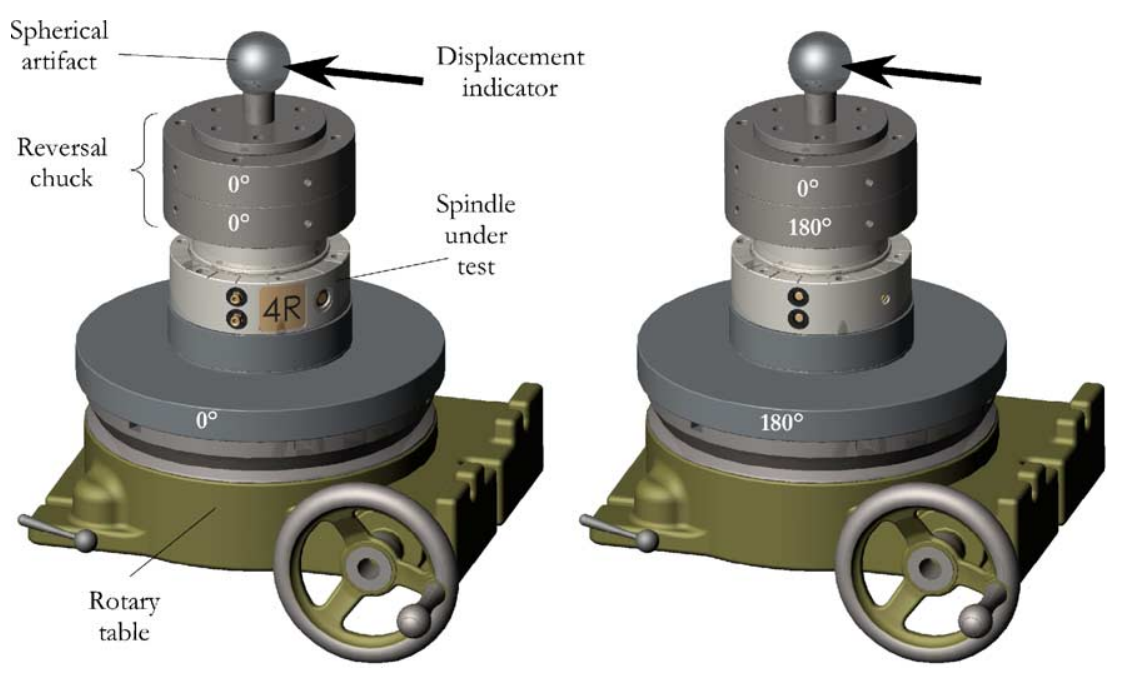

Fig. 3. Donaldson reversal using a rotary table to eliminate the need for relocating the displacement sensor.

a variety of metrology applications. Other types of precision spindles, including hydrostatic and rolling element, may be calibrated using the same techniques.

\subsection{Improved reversal implementation}

Donaldson and Estler reversal requires that two measurements be taken $180^{\circ}$ apart relative to the spindle stator. This is conventionally achieved by simultaneous measurements from two sensors or by moving one sensor between measurements. Either way, it is difficult to locate the sensor(s) exactly $180^{\circ}$ apart, at precisely the correct radial or axial locations, and at identical angles of incidence $\left(90^{\circ}\right)$.

A new implementation of Donaldson and Estler reversal was developed and investigated by Grejda that allows reversal to be executed without moving the displacement sensor [24]. In this implementation, illustrated in Fig. 3, the spindle under test is mounted on a rotary table, and a special chuck (referred to as a reversal chuck) is used between the spindle and artifact. The stator of the spindle under test is rotated with respect to the artifact and gage, rather than rotating the gage and artifact with respect to the spindle stator as done in the conventional method. A high quality rotary table can rotate the spindle stator with accuracy better than 10 arc-seconds, which makes this approach equally well suited for multi-position error separation. The reversal chuck, illustrated in Fig. 4, enables accurate $180^{\circ}$ reversal of the artifact using a pin in jig-ground indexing holes and accurate centering with a spherical pilot that mates in a carbide socket.

This simple but powerful improvement allows the procedure of calculating the spindle error motion at a particular location to be made with a single displacement sensor that is never moved. Rotating sensitive direction error motion may also be calculated by simply repeating the procedure with the rotary table oriented at $90^{\circ}$ and $270^{\circ}$.

\subsection{Sampling and signal processing}

The spindle measurements are processed in three steps. First, the data are anti-alias filtered, sampled and stored in equal angular increments as triggered by a rotary encoder mounted on the spindle's rotor. Second, a best fit, low order polynomial is removed from the data to reduce the influence of very low frequency disturbances such as thermal drift and air supply pressure fluctuations. Finally, the data are low-pass filtered in the Fourier domain to remove the fundamental component (when appropriate) and spectral content above the usable bandwidth of the instrument. The first and third steps are discussed in this section and the removal of low frequency disturbances is discussed separately in Section 3.3.

To prevent aliasing, the output from any analog displacement sensor must, without exception, be filtered with an ana$\log$ anti-alias filter prior to sampling by a data acquisition

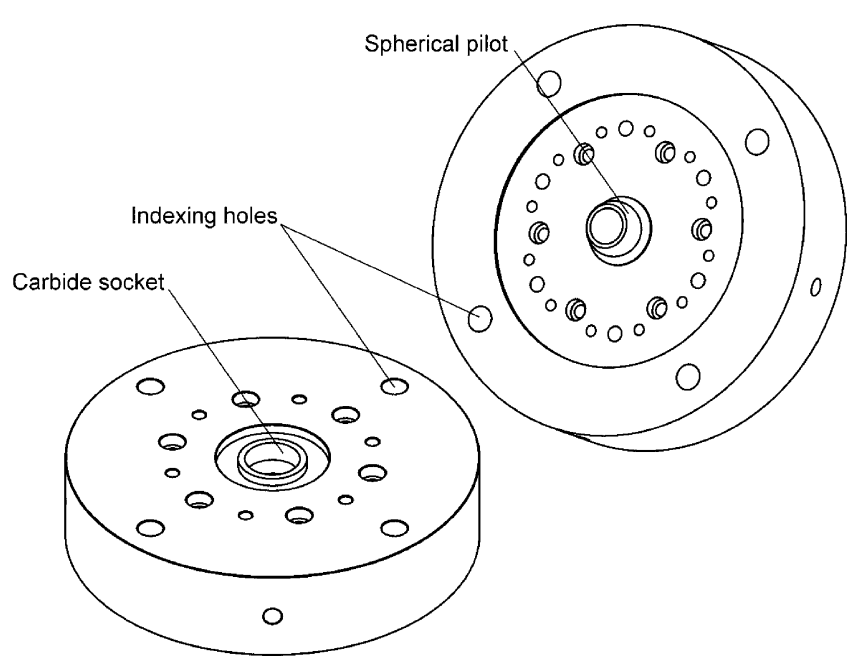

Fig. 4. The separated halves of the reversal chuck showing the precision ground mating surfaces and spherical pilot. 
system. In spindle metrology, the appropriate anti-alias cutoff frequency $f_{\text {cutoff }}$ depends on the number of encoder counts per revolution $c$ (typically a power of 2 such as 1024, 4096, or even higher) and the angular speed $n$ of the spindle as shown in Eq. (1). For our rotary encoder with 1024 counts per revolution, $f_{\text {cutoff }}$ must be lower than $8.5 n \mathrm{~Hz}$. In practice, the cutoff frequency is usually somewhat less to allow for the rolloff characteristics of realizable analog filters

$f_{\text {cutoff }}(\mathrm{Hz})=\frac{c \text { [counts per revolution] } \times n(\mathrm{rpm})}{2 \times 60}$.

An additional constraint on the usable bandwidth of an instrument is structural resonance. Spectral content above approximately one-third the first resonant frequency $f_{\mathrm{r}}$ is excessively amplified by resonance and should be removed. The first structural resonance of our instrument is $150 \mathrm{~Hz}$ in the sensing direction, which places a $50 \mathrm{~Hz}$ limit on bandwidth. The maximum allowable spindle speed during calibration $n_{\max }$ depends upon $f_{\mathrm{r}}$ and the desired cutoff $S$ as shown in Eq. (2). We use $S=50 \mathrm{cpr}$ (cycles per revolution) in our measurements, so the maximum spindle speed is $60 \mathrm{rpm}$

$n_{\max }(\mathrm{rpm})=\frac{60 f_{\mathrm{r}}(\mathrm{Hz})}{3 S(\mathrm{cpr})}$.

Once the data are properly anti-alias filtered and sampled, any additional low-pass filtering and removal of the fundamental component is carried out in the Fourier domain. There are two principal advantages to Fourier domain signal processing in spindle metrology. First, Fourier domain filtering causes no distortion on the data and precisely removes only the desired spectral components from the signal. Second, the synchronous and asynchronous components of spindle error motion are intuitively discernible in the Fourier domain. The synchronous component appears in the integer cpr Fourier bins, and the asynchronous component appears in the remaining, non-integer, Fourier bins. The non-integer bins of the asynchronous component are of special interest when measuring rolling element spindles because of the predictable frequencies at which defects appear.

Therefore, the measurements are low-pass filtered in the Fourier domain to remove spectral content above the desired cutoff frequency $S$. It is also convenient to remove the fundamental $(1 \mathrm{cpr})$ error motion in the Fourier domain for radial and face measurements. In both cases, the appropriate Fourier bins are set to zero on both sides of the Nyquist frequency. The fundamental error motion is removed by zeroing out the bin corresponding to the one cpr component and the corresponding bin on the mirror image of the Nyquist frequency. Similarly, the low-pass filter for limiting spectral content to $S$ is implemented by zeroing out the Fourier bins above $S$ and up to the mirror image of $S$ on the other side of the Nyquist frequency. The metrologist can quickly check the effect of running the data through low-pass filters with cutoffs such as 5,10 , and $50 \mathrm{cpr}$, just as is done with traditional roundness measuring instruments. Thompson describes the utility of this approach in more detail [25].
It is important to note that the Fourier domain representation of the data is considered in units of cpr rather than $\mathrm{Hz}$ since the data are synchronized to angle, not time. The range of cpr bins in the Fourier domain equals the number of encoder counts in a single revolution $c$. The resolution equals the inverse of the number of revolutions over which data are recorded. For example, if 32 revolutions of data are recorded, then the resolution is $1 / 32$ of a cpr. High resolution is helpful in resolving the closely spaced spectral content seen in ball bearing spindles.

After post-processing in the Fourier domain, an inverse discrete Fourier transform returns data to the angular domain, where error motion values are computed and the data are visualized using polar plots or the advanced techniques shown later in this paper.

\subsection{Thermal and air supply pressure fluctuations}

Although it is possible to minimize the sensitivity of a structure to variation in temperature through clever design and materials selection, some drift is still possible over short time intervals at the nanometer-level $[26,27]$. In the measurements performed for this article, the test duration was about $30 \mathrm{~s}$, and thermally induced drift varied from 0 to $5 \mathrm{~nm}$. Additional displacement fluctuations occurred due to variations in the regulated air pressure supplied to the air bearing spindles. Fig. 5 shows the low-frequency drift in the structural loop of the test hardware. The regular cycling of the HVAC system every $4.3 \mathrm{~min}$ is apparent, as is additional very low frequency drift due to other environmental effects.

Low frequency signals, like thermal drift and pressure fluctuations, which have periods exceeding the recording time of the spindle measurements, should be filtered from the measurements prior to a discrete Fourier transform. These low frequency signals do not necessarily have equal values at the beginning and end of the recorded data. This causes a well-known signal-processing phenomenon called leakage and is an artifact of the discrete Fourier transform since it assumes that the signal repeats indefinitely and that the test period captures an integer number of cycles. In other applications, leakage may be minimized through thoughtful use of windowing functions, but this introduces additional well-documented problems and should not be used for spindle metrology.

A more appropriate solution for removing low frequency drift and minimizing leakage in spindle calibration is to fit and remove a low order polynomial from each data set. In numerous tests with $30 \mathrm{~s}$ data captures it was found that a second-order polynomial fit the thermal drift data with its much longer period of oscillation.

\subsection{Description of hardware}

All results described in Section 4 were measured during the calibration of unmotorized air bearing spindles (Professional Instruments 4R) rotating at about $60 \mathrm{rpm}$ with a nom- 

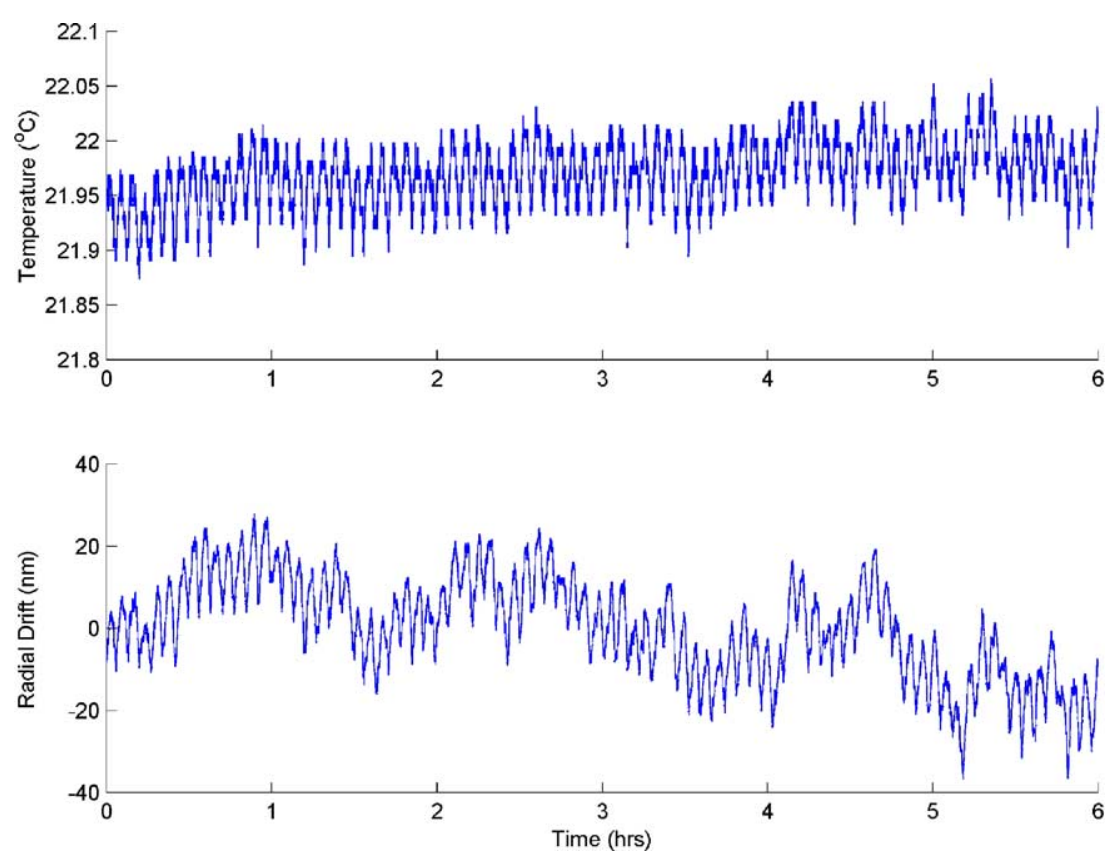

Fig. 5. Temperature variation and radial displacement drift over a six-hour interval.

inal air pressure of $830 \mathrm{kPa}$. A 1024 count encoder (Heidenhain ERO incremental) generated a pulse train for triggering and a once per revolution index pulse. The spindle under test is placed on a rotary table (Moore LRT) that is mounted on a three-axis measuring machine (Moore No. 3 Universal Measuring Machine). The three axes of travel allow convenient placement of the displacement sensor at the correct location and the rotary table eliminates the need to move the sensor between tests as described in Section 3.1. The spherical pilot maintains the centering of the artifact or master axis during reversal. The reversal chuck also features jig ground locating holes at $0^{\circ}, 90^{\circ}, 180^{\circ}$, and $270^{\circ}$ for accurate angular rotation in either fixed or rotating sensitive direction measurements.

Displacements are measured by capacitive sensors with $2 \mathrm{mV} / \mathrm{nm}$ sensitivity (Lion Precision DMT drivers with C1-C probes) with an internally adjustable analog filter set to a cutoff of $100 \mathrm{~Hz}$ for this testing. The analog output from the sensors is sampled by a 16-bit data acquisition card (National Instruments PCI-6110E). The data are subsequently analyzed using custom-designed software that executes the thermal drift removal, Fourier domain post processing and displays the results on an intuitive user interface [28]. The analysis of the data follows procedures described in Sections 3.2 and 3.3 as well as in the B89.3.4M standard.

\section{Experimental results}

In this section, four experiments demonstrate the ability to calibrate spindles with nanometer error motion. First, the repeatability of the techniques and apparatus are demonstrated. Second, the fixed sensitive direction synchronous radial error motion of two spindles is measured using the master axis method with sub-nanometer repeatability. Third, reproducibility of measurements using the different methods of Donaldson, multi-step and multi-probe error separation is demonstrated. Fourth, the results of a complete, five degree-of-freedom spindle calibration are shown using new

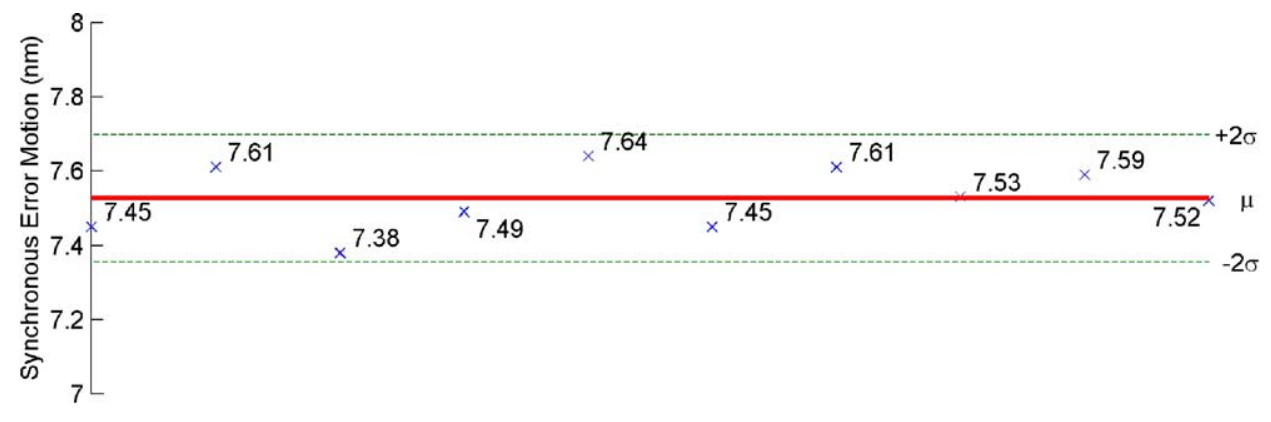

Fig. 6. Repeatability of 10 consecutive master axis measurements, without reversal. 


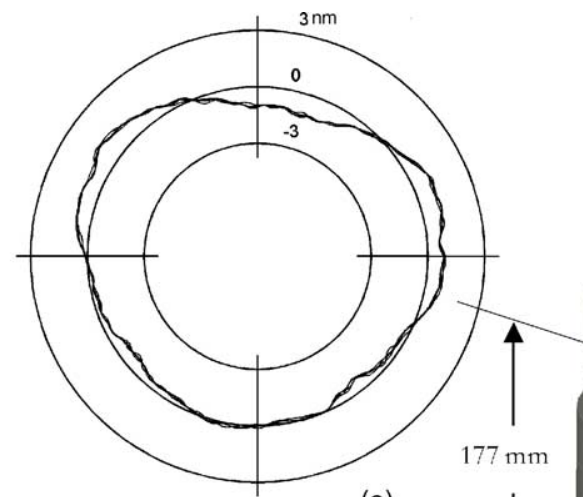

(a)

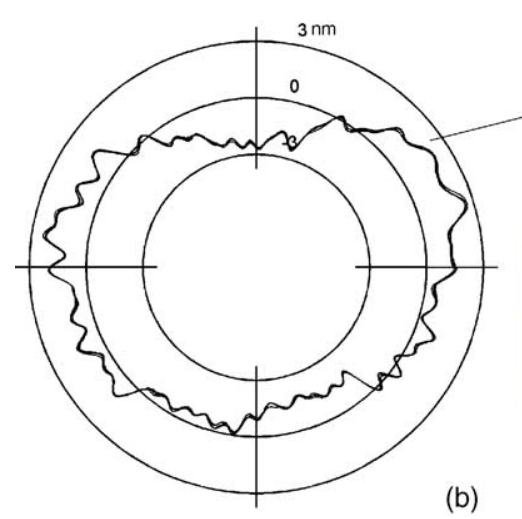

(b)

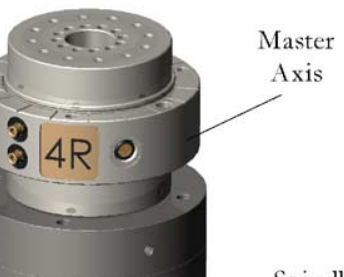

under test

Fig. 7. Synchronous radial error motion of (a) master axis spindle directly on stator center and (b) spindle under test 177 mm above stator center.

plotting techniques that enable quick interpolation of radial error motion at any axial location and face error motion at any radial location.

\subsection{Measurement repeatability without reversal}

The repeatability of the techniques and apparatus, excluding the reversal procedure, is first assessed with 10 independent master axis measurements over the course of 4 days. Since reversal was not performed, the results include the combined synchronous radial error motion of the spindle under test and the master axis. The bold line in Fig. 6 indicates a mean of $7.52 \mathrm{~nm}$. The dashed lines above and below the mean indicate \pm 2 standard deviations, and the standard deviation for these tests is $0.08 \mathrm{~nm}$. We therefore conclude that $95 \%$ of the measurements would fall within about $\pm 0.16 \mathrm{~nm}$ of the mean assuming a normal distribution.

\subsection{Measurement repeatability with reversal}

In a second experiment, the fixed sensitive direction synchronous radial error motion measurement by the master axis method was conducted four times, including error separation by Donaldson reversal. All four sets of measurement results for the spindle under test and master axis are shown in the polar plots of Fig. 7 and summarized in Table 1. The results demonstrate that the repeatability is less than $0.2 \mathrm{~nm}$ when
Donaldson reversal is included in the measurement procedure. This suggests that the new, rotary table implementation of Donaldson reversal does not significantly degrade the measurement repeatability.

To confirm the master axis results shown in Fig. 7, we compared the synchronous radial error motion as measured by the master axis method to results obtained using a spherical artifact having an out-of-roundness of $35 \mathrm{~nm}$. It is important to note that radial error motion results can only be compared if measured at the same axial location with respect to the spindle under test (now $254 \mathrm{~mm}$ above the center of the spindle under test). Both techniques yield the synchronous error motion value to within $1 \mathrm{~nm}$. Fig. 8 shows the close agreement using polar plots, and Table 2 summarizes the comparison.

Table 1

Results of four synchronous radial error motion measurements

\begin{tabular}{lll}
\hline Test & \multicolumn{2}{l}{ Radial error motion } \\
\cline { 2 - 3 } & Spindle under test $(\mathrm{nm})$ & Master axis spindle $(\mathrm{nm})$ \\
\hline 1 & 5.2 & 2.4 \\
2 & 5.4 & 2.4 \\
3 & 5.4 & 2.5 \\
4 & 5.2 & 2.4 \\
\hline
\end{tabular}

Fixed sensitive direction; radial error motion; 50 cpr cutoff; 32 revolutions of data; $60 \mathrm{rpm}$; axial position $=177 \mathrm{~mm}$ above bottom stator center; master axis method; includes reversal. 


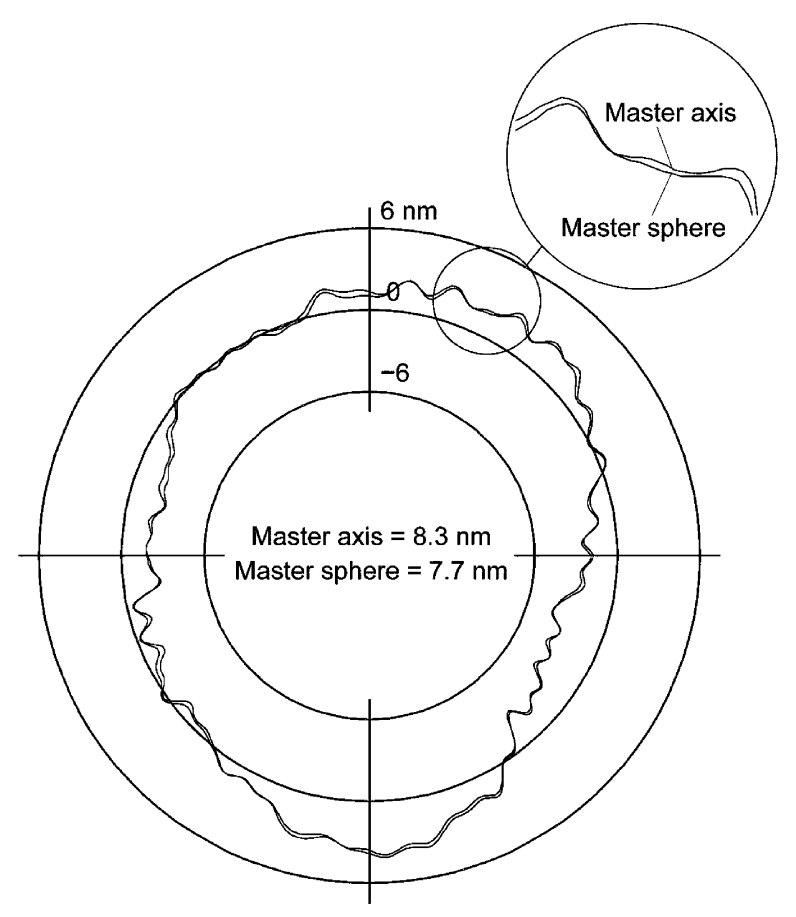

Fig. 8. Comparison of synchronous radial error motion of a spindle under test measured with a lapped spherical artifact and with a master axis.

\subsection{Measurement reproducibility by comparison of multi-step, multi-probe, and Donaldson reversal}

An additional advantage of mounting the spindle under test on a rotary table is that multi-probe and multi-step error separation techniques can also be performed without moving the displacement sensor. Therefore, we demonstrate reproducible measurements of synchronous radial error motion by comparing the results of alternative measurement methods. The fixed sensitive direction radial error motion and the form error of the lapped spherical artifact are compared using results obtained by Donaldson reversal, a 16-position multi-step test, and a 3-probe error separation test. The apparatus was completely disassembled between the tests.

As before, the tests were carried out on an unmotorized Professional Instruments air bearing spindle with $830 \mathrm{kPa}$ air pressure at $60 \mathrm{rpm}$ for 32 revolutions. The radial error mo-

Table 2

Comparison of radial error motion of a spindle under test using a master axis and a lapped artifact

\begin{tabular}{lll}
\hline Test method & \multicolumn{2}{l}{ Radial error motion } \\
\cline { 2 - 3 } & Synchronous (nm) & Asynchronous (nm) \\
\hline Master axis & 8.3 & 4.3 \\
Lapped artifact & 7.7 & 5.5
\end{tabular}

Fixed sensitive direction; radial error motion; 50 cpr cutoff; 32 revolutions of data; $60 \mathrm{rpm}$; axial position $=254 \mathrm{~mm}$ above stator center; Donaldson reversal. tion of the spindle is measured at an axial position $177 \mathrm{~mm}$ from the geometric center of the stator using a lapped spherical artifact as the capacitive displacement sensor's target. The Donaldson reversal was performed as described earlier.

The multi-step technique was performed using a step size of $22.5^{\circ}$ for a total of 16 measurements with an additional re-measurement of the $0^{\circ}$ position to check the repeatability with the first measurement. With the spindle on the rotary table, the multi-step procedure is readily carried out and requires only a bit of post-processing to perform the well-known calculations [23]. The form error of the artifact is determined from averaging the 16 measurements, and can be subtracted from the original measurement to compute the spindle error motion. Because of the 16 steps used in this measurement, the multi-step error separation method only distorts the Fourier components that are integer multiples of 16 cpr $(16,32,48 \mathrm{cpr}$, etc $)$.

The multi-probe method was carried out using the rotary table with only minor modifications to the approach documented in the literature [24]. For this study, the computations were performed using data collected for probes at $0^{\circ}, 122.5^{\circ}$, and the $202.5^{\circ}$ position (asymmetrically) to avoid masking the third harmonic of the error motion.

Fig. 9 shows the form error of the lapped spherical artifact as calculated by the three different methods. The form error results match closely with the exception of a deviation at approximately $160^{\circ}$. The agreement in form error is otherwise excellent, differing by less than $1 \mathrm{~nm}$. Additionally, the sphere's form error values measured by the three techniques agree to better than $1 \mathrm{~nm}$.

The fixed sensitive direction synchronous radial error motion of the spindle is also shown in Fig. 9. The Donaldson and multi-step results agree within a nanometer for the entire polar plot. However, the multi-probe results differ by $1.5 \mathrm{~nm}$ in some locations. This discrepancy may be attributed to the multi-probe method, which imperfectly computes the content of all frequency bins of the error motion during separation, rather than just specific harmonics as in the case of multi-step testing (integer multiples of 16 for the multi-step method used here).

Since the results agree within $1.5 \mathrm{~nm}$ for all three error separation methods, we conclude that the techniques and apparatus can reproducibly calibrate spindles at the nanometer level.

\subsection{Five degree-of-freedom characterization}

To this point, only fixed sensitive direction radial error motion at specific axial positions has been discussed. However, the accuracy of an object made by turning, facing, and general contouring is affected by radial, axial and tilt error motion of the axis of rotation used to generate it. The B89.3.4M standard outlines the procedure for making multiple measurements to determine the error motion at any axial or radial position. 

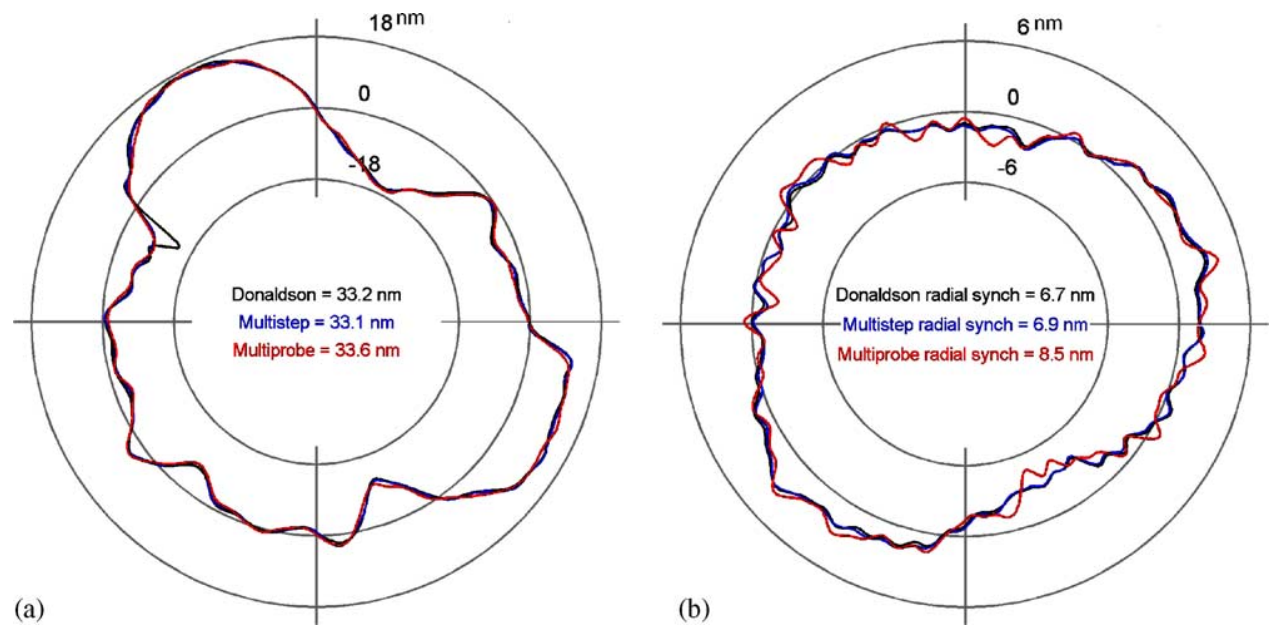

Fig. 9. Polar plots of artifact form error and synchronous radial error obtained by three error separation techniques. (a) Artifact form error, (b) synchronous radial error of spindle.

We performed five degree-of-freedom spindle calibrations using both the master axis method and the lapped double ball method with excellent agreement. The results shown in Figs. 10 and 11 use the master axis method with Donaldson reversal to compute radial and face error motion of the master axis and test spindles at any axial or radial position. Fig. 10 is the rotating sensitive direction radial error motion for axial positions up to $400 \mathrm{~mm}$ from the center of the stator of the spindle under test. On the right of the figure, slices (polar plots) show the synchronous radial error motion with values expressed in nanometers. The radial error motion at the center of this particular spindle is $2.2 \mathrm{~nm}$ with the tilt error motion contributing to slight increases further away from the center.

Fig. 11 shows the calculated rotating sensitive direction face error motion for radial positions ranging up to $400 \mathrm{~mm}$ from the $Z$-axis. These plots were prepared using data obtained by Estler face error motion reversal. Synchronous error motion slices are shown to the right of the figure for on axis and radial positions of 150 and $300 \mathrm{~mm}$. Two magnitudes are shown for the axial error motion measurement; the fundamental axial error motion is $1.1 \mathrm{~nm}$ and the residual synchronous axial error motion is $2.6 \mathrm{~nm}$. Again, the tilt error motion increases in magnitude moving radially outward

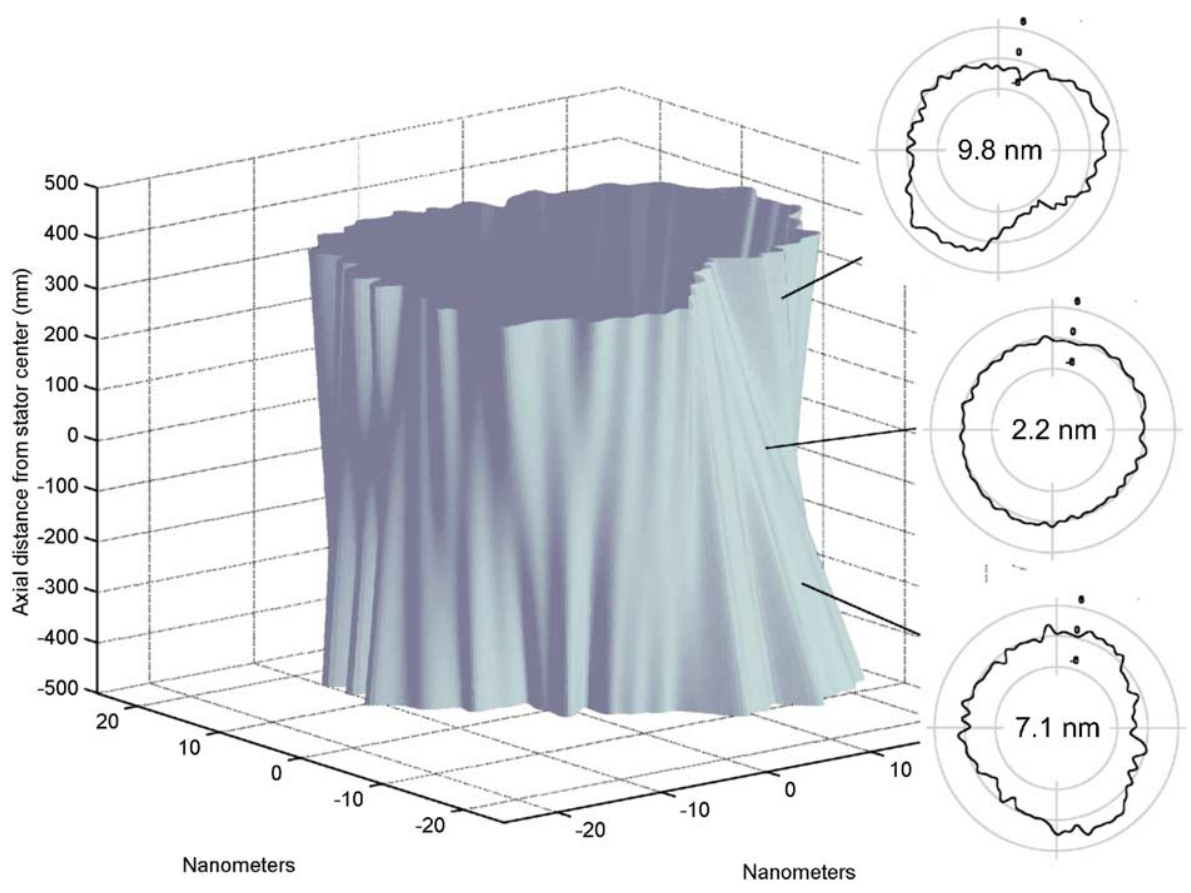

Fig. 10. Rotating sensitive direction synchronous radial error motion for various axial locations. 


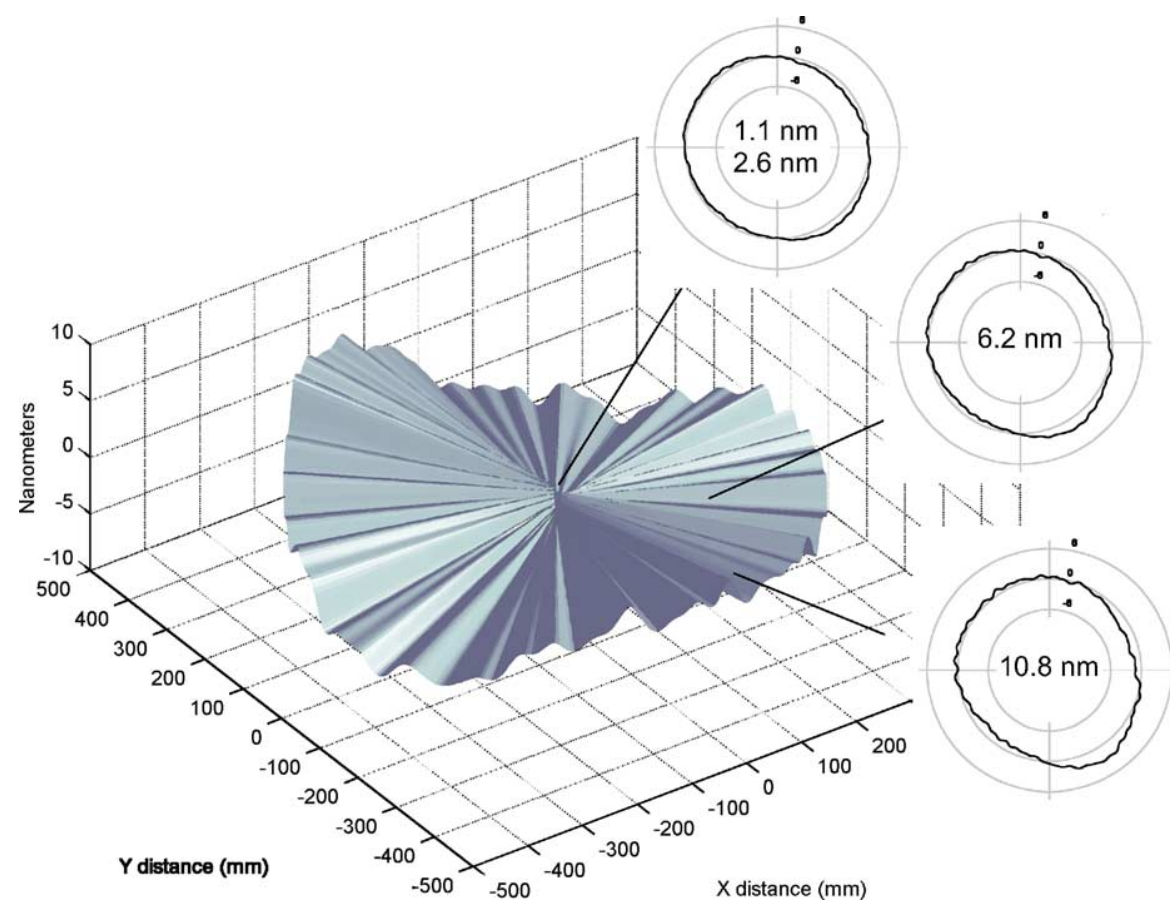

Fig. 11. Rotating sensitive direction synchronous face error motion for various radial locations.

from the axis of rotation, from $2.6 \mathrm{~nm}$ on-axis to $10.8 \mathrm{~nm}$ at $400 \mathrm{~mm}$.

\section{Conclusions}

This article demonstrates techniques for calibrating spindles with nanometer error motion. By using modern capacitive sensors, high quality data acquisition systems, thoughtfully designed structures, and appropriate signal processing techniques, it is now feasible to measure spindles with sub-nanometer repeatability and nanometer reproducibility. This was accomplished with a new implementation of Donaldson reversal and Estler reversal that uses a rotary table, a reversal chuck, and a stationary displacement sensor. This configuration eliminates any need to move the displacement sensor during reversal, and it also facilitates multi-step and multi-probe methods of error separation. For the first time, the master axis method was shown to provide results in agreement with lapped artifacts to less than $1 \mathrm{~nm}$.

\section{Acknowledgments}

The authors thank the National Science Foundation (DMI-9702897) for their support of this research.

\section{References}

[1] Bryan J, Clouser R, Holland E. Spindle accuracy. Am Mach 1967;149-164.

[2] Thompson DC. The design of an ultra-precision CNC measuring machine. CIRP 1988;38(1):501-4.
[3] Moore W. Foundations of Mechanical Accuracy. Bridgeport: Moore Special Tool Company, 1970.

[4] ANSI/ASME B89.3.4M Axes of Rotation: Methods for Specifying and Testing Standard, 1985

[5] Donaldson RR. A simple method for separating spindle error from test ball roundness error. CIRP 1972;21(1):125-6.

[6] Evans CJ, Hocken RJ, Estler WT. Self calibration: reversal, redundancy, error separation, and "absolute testing". CIRP 1996;45(2):61734.

[7] Zhang GX, Zhang YH, Yang ZL. A multipoint method for spindle error motion measurement. CIRP 1997;46(1):441-5.

[8] Mitsui K. Development of a new measuring method for spindle rotation accuracy by three points method. In: Proceedings of the 23rd International MTDR Conference; 1982. p. 115-21.

[9] Salsbury JG. Implementation of the Estler face motion reversal technique. Precis Eng 2003;27(2):189-94.

[10] Liebers MJ, Oss DD, Sheridan TP. The master axis method for spindle error motion calibration. In: Proceedings of the 13th ASPE Annual Meeting, Unpublished Poster Paper, St. Louis, Missouri; 1998.

[11] Marsh E, Grejda R. Experiences with the master axis method for measuring spindle error motion. Precis Eng 2000;24(1):50-7.

[12] Scheslinger G. Testing Machine Tools: for the use of Machine Tool Makers, Users, Inspectors, and Plant Engineers. 8th ed. New York: Pergamon Press; 1978.

[13] Tlusty J. Systems and methods of testing machine tools. Microtechnic 1959;13.

[14] Bryan JB, Vanherck P. Unification of terminology concerning the error motion of axes of rotation. CIRP 1975;24(2):555-62.

[15] Scientific Technical Committee Me. Unification document me: axes of rotation. CIRP 1976;25(2):545-64.

[16] Vanherck P, Peters J. Digital axis of rotation measurement. CIRP 1973;22(1):135-6.

[17] Arora GK, Mallanna C, Anantharaman BK, Babin P. Measurement and evaluation of spindle running error. Int $\mathrm{J}$ Mach Tool Des Res 1977;17(2):127-35.

[18] Murthy TSR, Mallanna C, Visveswaran ME. New methods of evaluating axis of rotation error. CIRP 1978;27(1):365-9. 
[19] Foldvari TL, Lion KS. Capacitive transducers. Instrum Contr Syst 1964.

[20] DeBra DB. Spindle metrology—a student Project. In: Presentation at the SME Seventh International Precision Machining and Gaging Symposium; 1986.

[21] Chapman PD. A capacitance based ultra-precision spindle analyzer. Precis Eng 1985;7(3):129-37.

[22] Hansen HJ. A New Dynamic Spindle Analyzer. LLNL PreprintUCRL 99490, 1988.

[23] Whitehouse DJ. Some theoretical aspects of error separation techniques in surface metrology. J Phys E: Sci Inst 1976;9:531-6.
[24] Grejda RD. Use and calibration or ultraprecision axes of rotation with nanometer level metrology. PhD Dissertation. Pennsylvania State University; 2002.

[25] Thompson DC. The compuron: how round is round? Energy Technol Rev 1982;1-9.

[26] McClure ER. Manufacturing accuracy through control of thermal effects. Ph.D. Thesis. UC, Berkeley; 1969.

[27] Bryan JB. International status of thermal error research. CIRP 1990;39(2):645-56.

[28] Vigliano VC. Computer control for precision bearing analysis. MS Thesis. Pennsylvania State University; 2001. 